\title{
躍Irestice
}

\section{Uma revisão integrativa sobre o uso de chatbot para subsidiar o ensino na área da saúde}

\author{
An Integrative Review on the Use of Chatbot to Support Health Education
}

\begin{abstract}
Jose Ricardo Vasconcelos Cursino ${ }^{1}$, Andrea Abreu Calista ${ }^{2}$, Jefferson Elder da Mota Nascimento ${ }^{3}$, Amadeu Sá de Campos Filho ${ }^{4}$
\end{abstract}

\begin{abstract}
Resumo
Introdução: O crescimento do uso de tecnologias educacionais se intensificou com o passar dos anos. Uma dessas tecnologias é o Chatbots, um Agente Inteligente de diálogo por meio de mensagens, em que seu uso na saúde já se mostrou promissor ao ser implementado em diversas áreas. Objetivo: Analisar a produção acadêmica de chatbots aplicados na educação em saúde, no período de 2013 à 2019. Método: Revisão integrativa, realizada a partir da combinação de 12 descritores, divididos em três domínios, em seis bases de dados, nas línguas portuguesa e inglesa. Resultados e discussão: Com 416 resultados iniciais, que após passar pelos critérios de inclusão e exclusão apresentaram-se em 31 publicações utilizadas nesta revisão. Após análise, notou-se que os principais usos do Chatbot são na educação (41,9\%) e na utilização como instrumento de apoio aos profissionais de saúde (22,5\%). Conclusão: Concluiu-se que a utilização de chatbots para o auxílio e o ensino aos pacientes, estudantes e profissionais é evidentemente eficaz, mas se faz necessária uma maior difusão dessa tecnologia com o objetivo de promover o uso mais efetivo dos chatbots.
\end{abstract}

Palavras-Chave: Educação, Saúde, Tecnologia Educacional.

\begin{abstract}
Introduction: The growth in the use of educational technologies has intensified as the years go by. One of these technologies are the Chatbots, an intelligent agent of dialogue through messages, its use in health has already proved promising as it is implemented in several areas. Objective: To analyze the academic production of chatbots applied in health education, from 2013 to 2019. Method: Integrative review, carried out from the combination of 12 descriptors, divided into 3 domains, into 6 databases, in Portuguese and English languages. Results and discussion: With 416 initial results, which after going through the inclusion and exclusion criteria resulted in 31 publications used in this review. After analysis, it was noted that the main uses of Chatbots are in education (41.9\%), and in using as an instrument to support professionals (22.5\%). Conclusion: It has concluded that the use of chatbots for the assistance and teaching of patients, students and professionals is effective but a greater dissemination of this technology is necessary in order to promote the more effective use of chatbots.
\end{abstract}

\footnotetext{
1 Graduando em Enfermagem. Centro Universitário São Miguel. Email: jricardo52525@gmail.com 2 Graduando em Medicina. Universidade Federal de Pernambuco. Email: andcalista@gmail.com

3 Graduando em Ciências da Computação. Universidade Federal de Pernambuco. Email: jemn@cin.ufpe.br

4 Doutor. Centro de Ciências Médicas. Universidade Federal de Pernambuco. Email: amadeu.campos@nutes.ufpe.br Correspondência: NUTES/UFPE - Hospital das Clínicas, 2ํandar. Av. Prof. Moraes Rego, no 1235, CEP: 50670-901 CDU Recife, PE
}

Rev. Saúde Digital Tec. Educ., Fortaleza, CE, v. 5, n. 1, p.108-122, jan./abr. 2020. 


\section{Introdução}

O crescimento exponencial do acesso à internet e sua incorporação às diversas atividades do cotidiano tem sido apontado como uma importante ferramenta para inclusão social e difusão de informações. No que tange à educação, novas tecnologias, tais como agentes inteligentes, chatbots e a inteligência artificial, podem ser definidas como um processo facilitador da integração entre teoria e prática, conhecimento e saber, e têm se apresentado como uma alternativa, para facilitar o processo de ensinoaprendizagem, tornando o usuário sujeito ativo do processo de construção do conhecimento ${ }^{1,2}$.

Entre as novas tecnologias existentes, sistemas que oferecem informações em tempo real, permitem que haja a interação com o conteúdo e admitem a obtenção de apoio durante o processo de aprendizado, propiciando não apenas a aquisição de conhecimentos, mas a construção de significações pessoais e o crescimento com a experiência do aprendizado ${ }^{3}$.

Diversas pesquisas, na área da saúde, elucidam a aplicabilidade dessas tecnologias para a aquisição de informações por usuários de diferentes faixas etárias, apontando ainda mais benefícios, tais como: a promoção de um ambiente interativo, a possibilidade de integração em redes sociais, a rapidez, facilidade, a privacidade fornecida, abrangência e possibilidade de promoção à saúde e auxílio na prevenção de diversas doenças ${ }^{4,5,6,7,8}$.

Observando essas necessidades, o uso dos Chatbots se torna o ideal. Por ser uma tecnologia que utiliza um banco de dados com o objetivo de fornecer informações de forma instantânea e que simule uma conversação por meio de aplicativos de mensagens, o uso dos chatbots se faz relevante. No meio médico essa já é uma prática existente e o auxílio mediado por essa tecnologia se encontra presente em diversas áreas, tais como: psiquiatria, clínica médica, saúde da mulher, dentre outras ${ }^{6,9,10}$.

Os pontos positivos citados acima tornam essa modalidade de ensino/aprendizagem convidativa para os mais variados grupos populacionais e para a abordagem dos mais diversos assuntos. Nesse sentido e, avaliando o cenário descrito, o artigo aqui apresentado traz o objetivo de realizar o levantamento bibliográfico das publicações sobre o uso de chatbots e dos Agentes Inteligentes como suporte para o aprendizado em saúde. Sendo assim exposto o panorama das publicações voltadas para este assunto, visou-se no presente trabalho, a exposição do tema no meio acadêmico 
como também, incentivar e nortear trabalhos futuros.

\section{Métodos}

Foi realizada uma revisão da literatura de forma integrativa utilizando três áreas domínios: agente, utilização e área de atuação. Para cada área foram escolhidos os descritores em inglês e português com seus sinônimos totalizando 12 descritores distribuídos da seguinte forma: agente (chatbot, artificial intelligence, conversational agent, relational agent, embodied conversational agent, virtual agent), propósito (education, training, simulation) e área de atuação (nursing, medicine e healthcare).

Os critérios de inclusão utilizados nesta pesquisa são: Chatbot como tecnologia educacional para o ensino de um conteúdo específico no curso de saúde, publicado nos últimos sete anos, conter metodologia definida, ser uma pesquisa de abordagem qualitativa ou quantitativa, e ser disponibilizada nos idiomas inglês, português e espanhol. E como critérios de exclusão: abordagem do chatbot de maneira geral (revisão/levantamento); não ter a metodologia descrita; não ser possível a obtenção do texto completo.

A pesquisa foi realizada em seis bases de dados: PUBMED, IEEE, BDENF, SCIELO, LILACS e SCI nos últimos sete anos, de 2013 a 2019. Inicialmente, foram obtidos um total de 416 resultados e, após uma análise inicial, 90 artigos estavam duplicados e foram excluídos resultando em 326 publicações que passaram para próxima fase. Durante a segunda etapa da revisão foi realizada a leitura do título e do resumo dos artigos. No final da leitura foi eliminado um total de 292 publicações por não se enquadrarem nos critérios de inclusão na pesquisa. Já nas 34 publicações restantes, foi realizada a leitura do texto completo, porém, nesta fase foram ainda eliminadas três publicações pois o texto completo não estava disponível, conforme demonstrado na figura 1. Foi utilizado então um total de 31 publicações, sendo 29 artigos e duas dissertações de mestrado.

\section{Resultados}

Foram encontrados 31 artigos que se enquadraram nos critérios de inclusão do presente estudo. Inicialmente classificamos as publicações em relação aos países de origem da produção. Essa análise foi importante tanto para identificar onde tem ocorrido maior produção de chatbots e de agentes de inteligência artificial por inferir o nível de produção tecnológica do país, como o porquê de diferentes países poderem apresentar realidades e, consequentemente, necessidades de intervenções diferentes, inclinando os objetivos da produção científica para a resolução de problemas encontrados naquela região.

\subsection{País de publicação.}


Ao agrupar os artigos por país de publicação se obteve o expressado no gráfico 1 (Nos anexos). Dentre os artigos encontrados na pesquisa realizada, o país que mais produziu durante esses últimos sete anos foi os Estados Unidos da América, representando 29\% ( $\mathrm{N}=31)$ das publicações coletadas e, em sequência, temos a China como segundo maior produtor. Nossos dados estão em conformidade com pesquisas internacionais na área de Inteligência Artificial, como o relatório produzido pela Editora Elsevier em dezembro de 2018, que relata a China e Estados Unidos da América como os territórios que concentram maior número de publicações da área ${ }^{20}$, como podemos observar na figura 2 (anexos).

\subsection{Ano de publicação.}

Ainda observando a Figura 2, podese notar o alto número de produções no ano de 2017 quando comparado aos anos anteriores e posteriores, especialmente nos territórios que concentram a maior quantidade de publicações. Esse aumento se deve ao avanço continuado no desenvolvimento de novas tecnologias que permitem o aprimoramento dos sistemas de Inteligência Artificial tornando-os cada vez mais rápidos e ampliando o campo de aplicações práticas dos sistemas.

Esse crescimento também foi identificado em nossa pesquisa, em que mais de $48 \%$ das publicações $(\mathrm{N}=31)$ ocorreram no ano de 2017, como podemos visualizar no gráfico 2 (Nos anexos).

\subsection{Público alvo.}

Outro tipo de variável escolhida para se analisar o conteúdo dos artigos encontrados foi o público-alvo das publicações. $\mathrm{O}$ resultado dessa distribuição encontra-se no gráfico 3 (Nos anexos).

Como observado no gráfico 3 (Nos anexos), o paciente foi o principal alvo das pesquisas publicadas, com $45,16 \%(\mathrm{~N}=31)$ das publicações, em sequência temos os profissionais e os estudantes das diversas áreas da saúde, ambos com 19,3\% $(\mathrm{N}=31)$, estando os desenvolvedores de agentes inteligentes em seguida, 16,1\% $(\mathrm{N}=31)$.

O foco no paciente tem aumentado devido o surgimento da internet, à crescente busca da população por conhecimento acerca de sua saúde e o constante déficit de profissionais disponíveis no momento para sanar essa necessidade. Além disso, o crescimento de pesquisas sobre a temática em sites que tendem a trazer informações generalizadas ao pesquisador e que podem o levar a conclusões equivocadas em relação ao seu estado de saúde ou condutas apropriadas para sua condição clínica ${ }^{6,912,13}$.

\subsection{Assuntos abordados.}




\section{Artigos destinados a pacientes}

Nos artigos destinados aos pacientes, houve uma grande variedade de temas da saúde sendo abordados. Esses temas podem ser visualizados no gráfico 4 (Nos anexos).

Podemos notar que o tema mais utilizado foi a prevenção precoce na saúde dos pacientes $(71,5 \% \mathrm{~N}=14)$. Isso pode ser notado pelo número de publicações voltadas para: a promoção de saúde, sendo representadas pelas orientações que visam modificar os hábitos de vida do paciente, estando nessa categoria a adoção de hábitos mais saudáveis como a prática regular de atividade física; a Educação em saúde com artigos que buscavam um agente capaz de tirar dúvidas sobre doenças diversas, bem como trazer informações sobre patologias específicas e tentando tornar o paciente mais orientado para participar ativamente da tomada de decisões junto ao profissional médico ${ }^{29,30}$; e a Prevenção de doenças específicas, como o câncer de colo uterino ${ }^{31}$.

Merece destaque ainda os artigos voltados para melhorar a adesão do paciente à terapia prescrita $(14,3 \% \mathrm{~N}=14)$ visto que mais de $50 \%$ dos pacientes portadores de doenças crônicas que necessitam de tratamento por períodos prolongados, como exemplo a tuberculose, apresentam dificuldades para manterem a assiduidade no tratamento. Os principais obstáculos dessa dificuldade são a grande quantidade de medicamentos, os efeitos adversos e o alto custo. Desta forma, um agente de conversação que interage com o paciente durante 24 horas por dia, programado para lembrar os horários de uso, prestar esclarecimentos sobre importância do tratamento e monitorar efeitos adversos relatados, pode conseguir maior adesão do paciente à terapia do que um profissional que de saúde que terá contato com este paciente apenas em consultas periódicas ${ }^{15}$.

\section{Artigos destinados aos estudantes}

O segundo público alvo avaliado foram os estudantes de graduação que, representa $19,3 \% \quad(\mathrm{~N}=31) \quad$ do total encontrado, o que em números se expressa em seis publicações, divididas conforme o gráfico 5 (Nos anexos). De acordo com o assunto abordado, esses artigos podem ser classificados em: cinco deles são direcionados ao treinamento da prática profissional futura, dos quais quatro $^{2,14,17,19}$ na atuação do diagnóstico e nas condutas profissionais frente as patologias e $u^{21}$ visando melhorar a comunicação entre o futuro profissional e seu paciente; $O$ sexto artigo ${ }^{22}$ tem como foco na reflexão e no incentivo a pesquisa na área de tecnologia, demonstrando avanços, usos e potencialidade do uso de chatbots na saúde.

Sabendo que o número de processos por erros médicos têm aumentado nas últimas décadas e devido 
à repercussão dos casos na mídia, a prevenção do erro é um dos grandes objetivos para o uso das tecnologias na prática em saúde e o treinamento com agentes virtuais de aprendizado surgem como uma alternativa segura, de fácil utilização e avaliação, tornando-se uma excelente ferramenta para a capacitação de profissionais ${ }^{14,16,17}$.

Levando em consideração a necessidade de tais ferramentas, Lima ${ }^{14}$, traz uma solução para o problema: a criação de um Serious Game em 3D onde possui um chatbot com o objetivo de fornecer treinamento à prática profissional sem intercorrências ou erros médicos.

\section{Artigos destinados aos profissionais.}

O terceiro público alvo avaliado foram os Profissionais da saúde, que semelhantemente aos estudantes detêm $19,3 \%(N=31)$ dos dados encontrados, o que expressa seis publicações que estão, por sua vez, divididas em áreas específicas conforme pode ser visualizado no gráfico 6 (nos anexos).

Em relação aos objetivos dos artigos, os seis artigos encontrados utilizaram os agentes inteligentes com 0 intuito de melhorar a assistência médica: sendo um deles voltado para simular um integrante da equipe de profissionais, avaliando quão bem seria a integração e a tomada de decisão com o auxílio do agente durante procedimentos cirúrgicos ${ }^{23}$; Em outros três artigos, o Agente Inteligente auxilia os profissionais no momento de suas consultas, sendo na interação com pacientes portadores do Transtorno do Espectro Autista (TEA), como na confecção de diagnósticos, além do aconselhamento a pacientes psiquiátricos fornecendo aos profissionais dados para a implementação de um tratamento mais eficaz $^{11,24,25}$. Por fim, os dois artigos restantes, são voltados para análise das opiniões dos profissionais a respeito da usabilidade dos chatbots durante 0 exercício profissional ${ }^{12,27}$.

Quando avaliado a opinião de médicos sobre a utilização de chatbots, Palanica $^{12}$, relata que:

"Os médicos acreditam nos custos e benefícios associados aos chatbots, dependendo da logística e dos papéis específicos da tecnologia. As áreas onde os médicos acreditavam que chatbots seriam mais úteis foram na melhoria da nutrição, dieta e adesão ao tratamento, bem como tarefas logísticas, como agendamento de consultas, localização de clínicas e fornecimento de lembretes de medicação. Os principais desafios percebidos foram a incapacidade dos chatbots de compreender as emoções e abordar toda a extensão das necessidades do paciente."

Ainda segundo a autora, o principal risco elencado pelos entrevistados foi a imprecisão das informações fornecidas pelo chatbot avaliado. Foi salientado ainda pela autora que a "automação logística simples" seria um ponto positivo para a utilização dos chatbots ${ }^{12}$.

\section{Artigos destinados a desenvolvedores.}


Por fim, o gráfico 7 (nos anexos) apresenta os assuntos abordados nos artigos que possuem como alvo os desenvolvedores de Agentes Inteligentes. Os artigos tratam especificamente dos métodos de criação e aprimoramento desses sistemas. O objetivo desses artigos é comparar diferentes formas de produção ou testar uma nova metodologia buscando construir um agente mais eficiente.

Como podemos observar no gráfico 7 (nos anexos), dois artigos têm como preocupação o desenvolvimento de um agente capaz de identificar palavras inseridas pelo usuário que possam indicar sentimentos ou mudança de sentimentos de um estado basal já associado àquele usuário. Esse fato acontece porque um dos objetivos pretendidos pelos desenvolvedores consiste em tornar a interação com o chatbot o mais próximo possível de uma interação com um humano. Em contrapartida um dos principais obstáculos é torná-lo sensível ao reconhecimento de emoções e sentimentos $^{7,18}$.

Dos demais artigos da categoria, outros dois ${ }^{20,8}$ têm como objetivo desenvolver modelos de recuperação de resposta mais rápido e eficiente, de forma que durante a captação das palavras chaves fornecida pelo usuário o sistema encontre no banco de dados a resposta mais adequada e no menor tempo possível. O último dos artigos tem como proposta criar um banco de dados de fontes diversificadas para que o sistema tenha mais opções de respostas adequadas de forma rápida e sem sobrecarga do sistema ${ }^{28}$.

Podemos observar com isso que os artigos destinados a desenvolvedores têm em comum a busca pelo desenvolvimento de um agente que adquira ao máximo, características de interação social humana, tendo em vista que uma das principais queixas de usuários de sistemas de inteligência artificial têm relação a automaticidade das aplicações como, por exemplo, aplicações com a voz robotizada (quando as tecnologias utilizam interação por meio de voz), tempo para a obtenção da resposta e a grande quantidade de respostas inapropriadas para questionamento levantado pelo usuário $8,18,19$.

\section{Conclusão}

O conjunto dos artigos coletados nessa revisão revelou alguns pontos que retratam a realidade das pesquisas utilizando chatbot, mesmo sendo uma tecnologia criada nos anos 60 a sua criação. A sua aplicação na área da saúde é a mais recente, tendo uma expressiva impressão em 2017 conforme pode ser visto no gráfico 2 (nos anexos). O público alvo que foi contemplado com maior quantidade de trabalhos, foi 0 representado pelos pacientes, gráfico 3 (nos anexos); nas diversas áreas de atuação como por exemplo, as áreas de 
educação e de incentivo a melhoria de hábitos de vida, gráfico 4 (nos anexos).

Esses dados coletados revelam também que o chatbot, mesmo sendo um meio pouco utilizado, é na verdade uma tecnologia com grande facilidade de uso, de criação e de alcance ao público devido a atual difusão dos meios tecnológicos na sociedade. Elencado esses pontos se faz necessário uma difusão maior dessas tecnologias, visando aplicabilidade nos diversos setores da saúde, trazendo assim melhorias que serão de grande importância para o meio acadêmico e social.

\section{Referências}

1. Su $\mathrm{MH}, \mathrm{Wu} \mathrm{CH}$, Huang $\mathrm{KY}$, Hong QB, Wang HM. A chatbot using LSTMbased multi-layer embedding for elderly care. In: Proceedings of the 2017 International Conference on Orange Technologies, ICOT 2017. 2018.

2. Melo ECA de, Enders BC, Basto ML. Plataforma PEnsinar $\AA$ : a learning tool for teaching the nursing process. Revista Brasileira de Enfermagem. 2018.

3. King AC, Campero I, Sheats JL, Castro Sweet CM, Garcia D, Chazaro A, et al. Testing the comparative effects of physical activity advice by humans vs. computers in underserved populations: The COMPASS trial design, methods, and baseline characteristics. Contemp Clin Trials. 2017;

4. Goldenthal SB, Portney D, Steppe E, Ghani K, Ellimoottil C. Assessing the feasibility of a chatbot after ureteroscopy. mHealth. 2019.

5. Magnani JW, Schlusser CL, Kimani E, Rollman BL, Paasche-Orlow MK, Bickmore TW. The Atrial Fibrillation Health Literacy Information Technology System: Pilot Assessment. JMIR Cardio. 2017;
6. Wu Y, Samant D, Squibbs K, Chaet A, Morshedi B, Barnes LE. Design of interactive cancer education technology for Latina farmworkers. In: 2014 IEEE Systems and Information Engineering Design Symposium, SIEDS 2014. 2014. 7. Wang $\mathrm{H}$, Zhang $\mathrm{Q}$, Ip M, Fai Lau JT. Social Media-based Conversational Agents for Health Management and Interventions. Computer (Long Beach Calif). 2018;

8. Zhu Z, Liu X, Li H, Li T. UB-CQA:

$A$ user attribute based community question answering system. In:

Proceedings of the 2017 12th International Conference on Intelligent Systems and Knowledge Engineering, ISKE 2017. 2018.

9. Owens OL, Felder T, Tavakoli AS, Revels AA, Friedman DB, Hughes-Halbert $C$, et al. Evaluation of a Computer-Based Decision Aid for Promoting Informed Prostate Cancer Screening Decisions Among African American Men: iDecide. Am J Heal Promot. 2019; 10. Jack B, Bickmore T, Hempstead M, Yinusa-Nyahkoon L, Sadikova E, Mitchell S, et al. Reducing Preconception Risks Among African American Women with Conversational Agent Technology. J Am Board Fam Med. 2015;

11. Tielman ML, Neerincx MA, Van Meggelen M, Franken I, Brinkman WP. How should a virtual agent present psychoeducation? Influence of verbal and textual presentation on adherence.

Technol Heal Care. 2017;

12. Palanica A, Flaschner $P$, Thommandram A, Li M, Fossat Y. Physicians' perceptions of chatbots in health care: Cross-sectional web-based survey. J Med Internet Res. 2019;

13. Gardiner PM, McCue KD, Negash LM, Cheng T, White LF, Yinusa-Nyahkoon $L$, et al. Engaging women with an embodied conversational agent to deliver mindfulness and lifestyle recommendations: A feasibility randomized control trial. Patient Educ Couns. 2017;

14. De Lima RM, De Medeiros Santos A, Neto FMM, De Sousa Neto AF, Leao FCP, De Macedo FT, et al. A 3D serious game for medical students training in clinical cases. In: 2016 IEEE International 
Conference on Serious Games and Applications for Health, SeGAH 2016. 2016.

15. Guhl EN, Schlusser CL, Henault LE, Bickmore TW, Kimani E, PaascheOrlow MK, et al. Rationale and design of the Atrial Fibrillation health Literacy Information Technology Trial: (AF-LITT). Contemp Clin Trials. 2017;

16. Burke SL, Bresnahan T, Li T, Epnere K, Rizzo A, Partin M, et al. Using Virtual Interactive Training Agents (ViTA) with Adults with Autism and Other Developmental Disabilities. J Autism Dev Disord. 2018;

17. Kron FW, Fetters MD, Scerbo MW, White CB, Lypson ML, Padilla MA, et al.

Using a computer simulation for teaching communication skills: A blinded multisite mixed methods randomized controlled trial. Patient Educ Couns. 2017;

18. Meropi $P$, Billis AS, Hasanagas

ND, Bratsas C, Antoniou I, Bamidis PD.

Conditional Entropy Based Retrieval Model in Patient-Carer Conversational Cases. In: Proceedings - IEEE

Symposium on Computer-Based Medical Systems. 2017.

19. Epstein JH, Levin M, Jowell MS. Agent based simulation for training and assessing students in the field of anesthesiology. In: Proceedings of CBMS 2013 - 26th IEEE International Symposium on Computer-Based Medical Systems. 2013.

20. Denecke K, Tschanz M, Dorner $\mathrm{TL}$, May R. Intelligent conversational agents in healthcare: Hype or hope? In: Studies in Health Technology and Informatics. 2019.

21. Rivera-Gutierrez D, Kleinsmith A, Johnson T, Lyons R, Cendan J, Lok B.

Towards a reflective practicum of embodied conversational agent experiences. In: Proceedings - IEEE 14th International Conference on Advanced Learning Technologies, ICALT 2014. 2014.

22. Meskó B, Hetényi G, Gyorffy Z. Will artificial intelligence solve the human resource crisis in healthcare? BMC Health Services Research. 2018.

23. Lok B, Chuah JH, Robb A, Cordar A, Lampotang S, Wendling A, et al. Mixed-
Reality Humans for Team Training. IEEE Comput Graph Appl. 2014;

24. Madhu D, Jain CJN, Sebastain E, Shaji S, Ajayakumar A. A novel approach for medical assistance using trained chatbot. In: Proceedings of the International Conference on Inventive Communication and Computational Technologies, ICICCT 2017. 2017. 25. Lee D, Oh KJ, Choi HJ. The chatbot feels you - A counseling service using emotional response generation. In: 2017 IEEE International Conference on Big Data and Smart Computing, BigComp 2017. 2017.

26. Oh KJ, Lee D, Ko B, Choi HJ. A chatbot for psychiatric counseling in mental healthcare service based on emotional dialogue analysis and sentence generation. In: Proceedings - 18th IEEE International Conference on Mobile Data Management, MDM 2017. 2017.

27. Huijnen CAGJ, Lexis MAS, Jansens R, de Witte LP. Roles, Strengths and Challenges of Using Robots in Interventions for Children with Autism Spectrum Disorder (ASD). J Autism Dev Disord. 2019;

28. Jiang $R$, Banchs RE, Kim S, D'Haro LF, Niculescu Al, Yeo KH. Configuration of dialogue agent with multiple knowledge sources. In: 2015 Asia-Pacific Signal and Information Processing Association Annual Summit and Conference, APSIPA ASC 2015. 2016.

29. Mansilla A, Ponce J, Hernández A, Ochoa A, Herrera M, Cossio E. Implementation of an Chatbot in a Serious Game associated with the acquisition of social skills and the promotion of collaborative tasks in children. In: 12th Latin American Conference on Learning Objects and Technologies, LACLO 2017. 2017.

30. Kasinathan V, Xuan FS, Wahab MHA, Mustapha A. Intelligent Healthcare Chatterbot (HECIA): Case study of medical center in Malaysia. In: 2017 IEEE Conference on Open Systems, ICOS 2017. 2018.

31. Gardiner PM, McCue KD, Negash LM, Cheng T, White LF, Yinusa-Nyahkoon 
$L$, et al. Engaging women with an embodied conversational agent to deliver mindfulness and lifestyle recommendations: A feasibility randomized control trial. Patient Educ Couns. 2017; 


\section{Anexos}

Figura 1 - Fluxo de obtenção dos artigos segundo os critérios metodológicos.

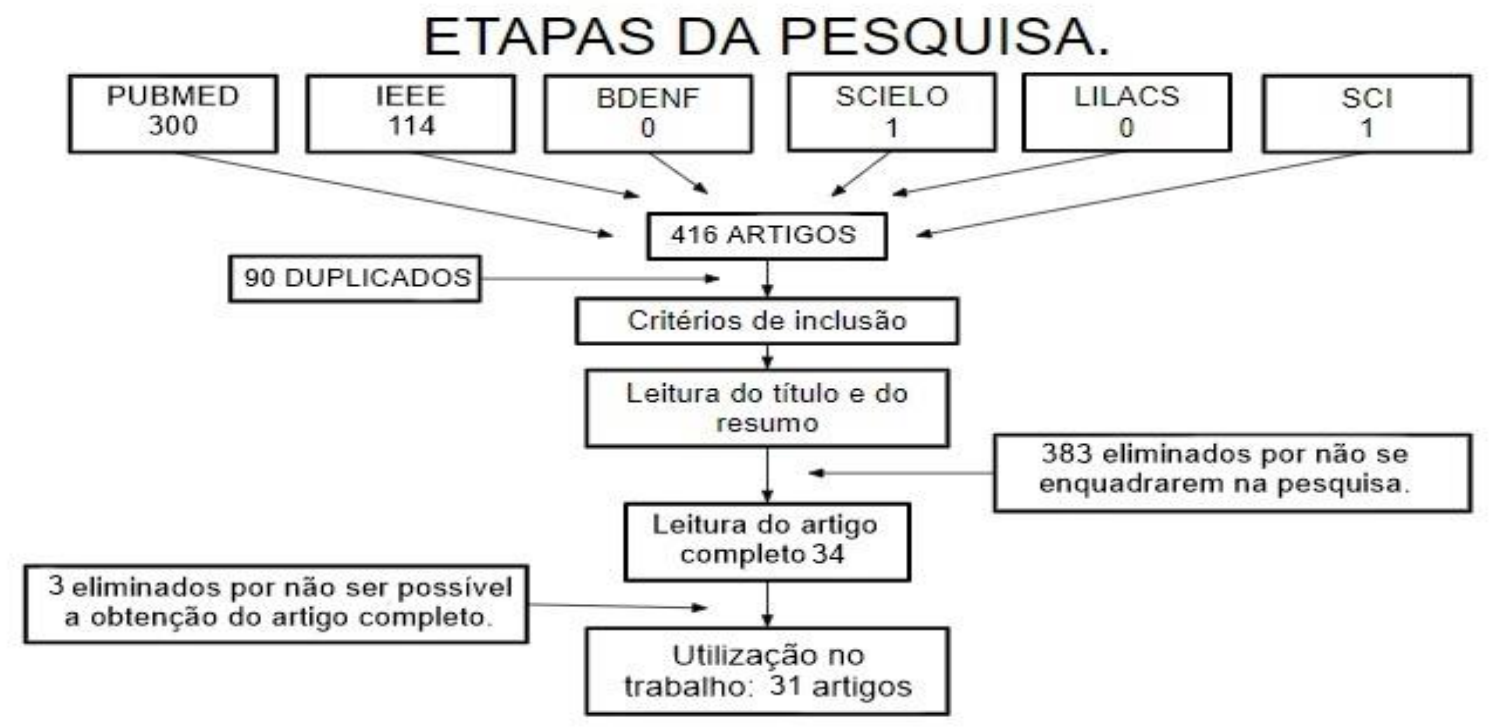

Fonte: os autores.

Figura 2 - Publicações por país/território sobre Chatbots e Agentes Inteligentes em 05 anos (2013- 2017).

\section{Number of publications in Al}

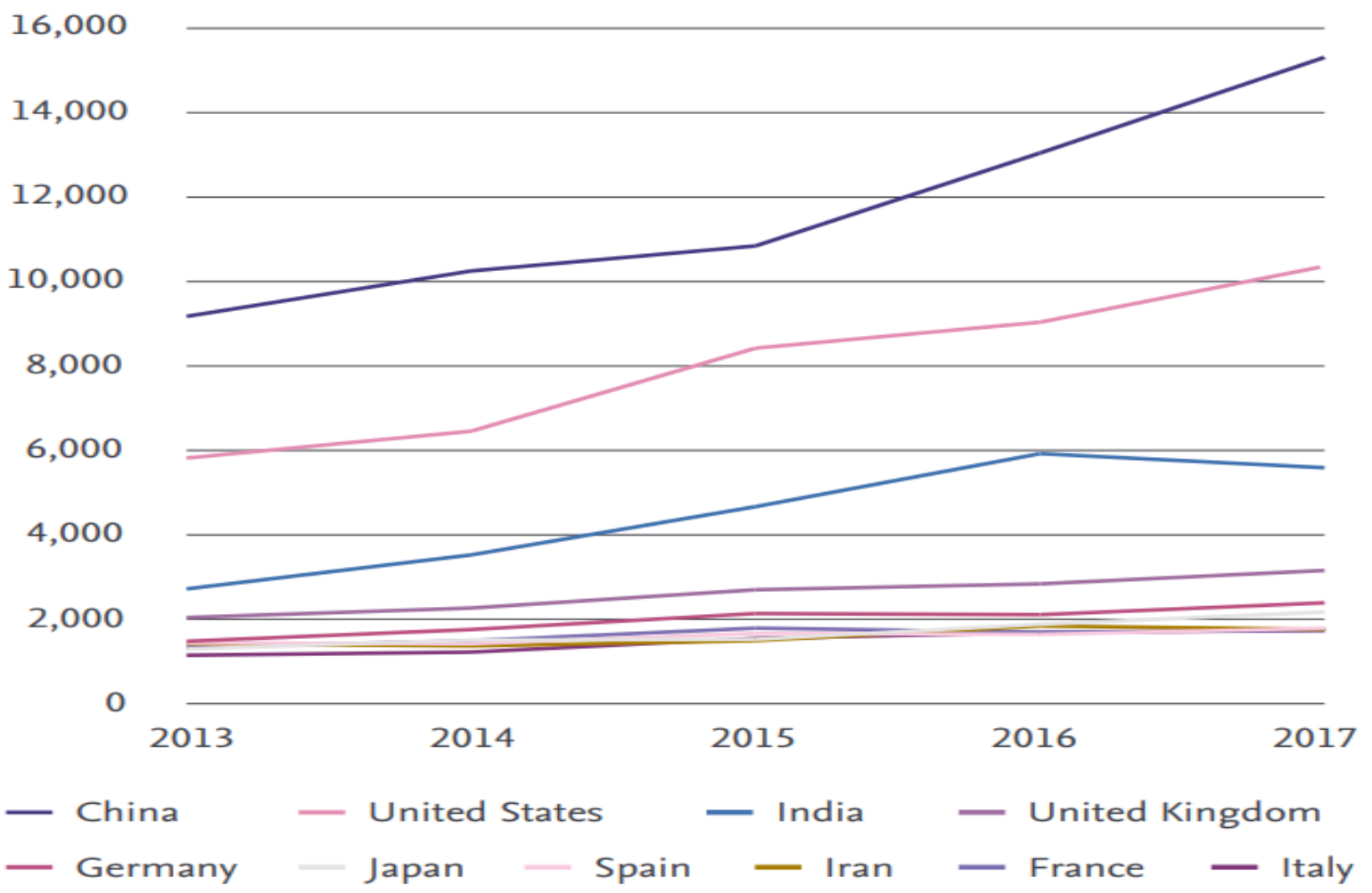

Fonte: Scopus In: Annoni et al., 2018. ${ }^{20}$ 
Gráfico 1 - Distribuição dos artigos selecionados por país de publicação.

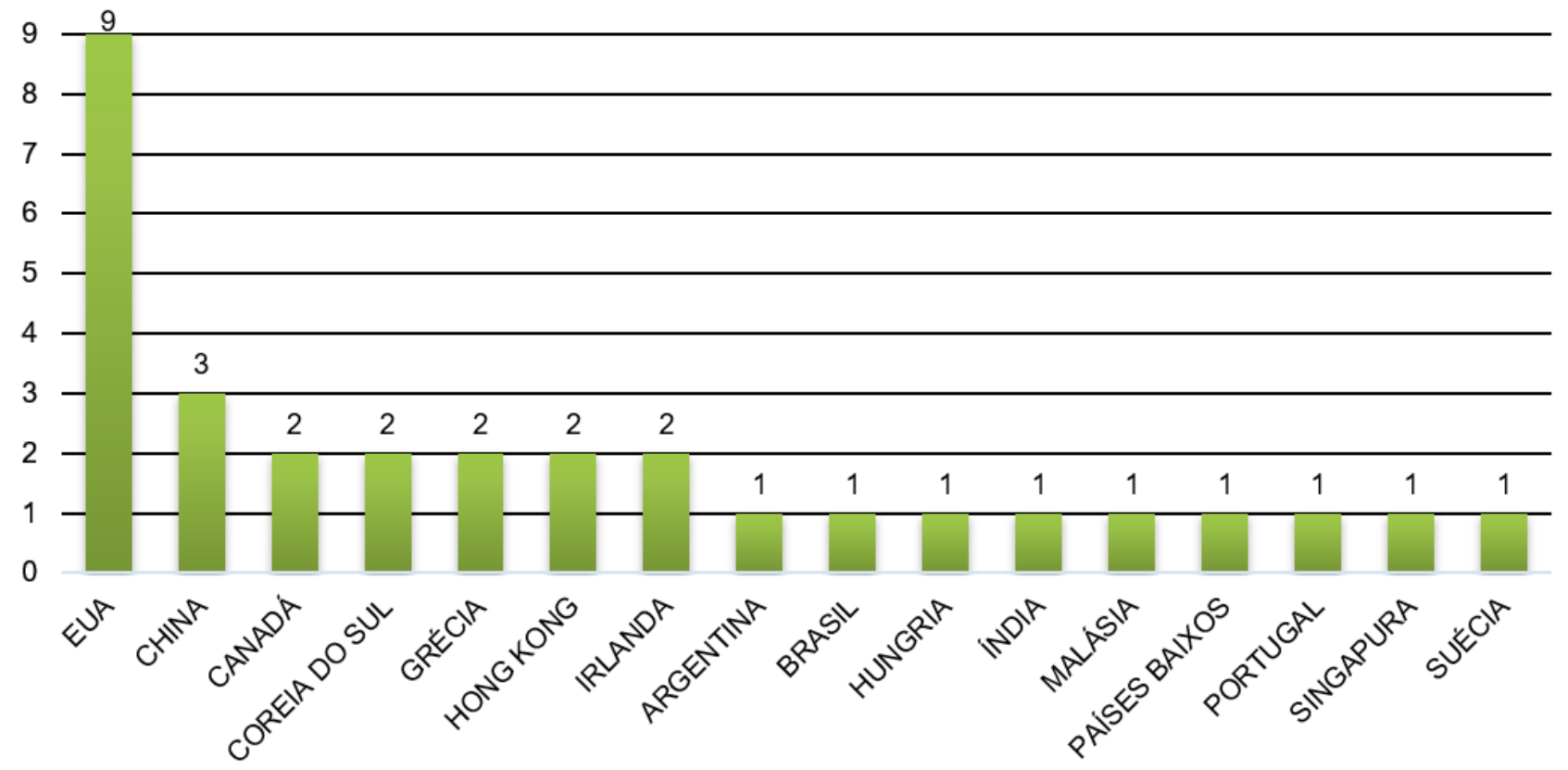

Fonte: os autores.

Gráfico 2 - Distribuição dos artigos selecionados por ano de publicação.

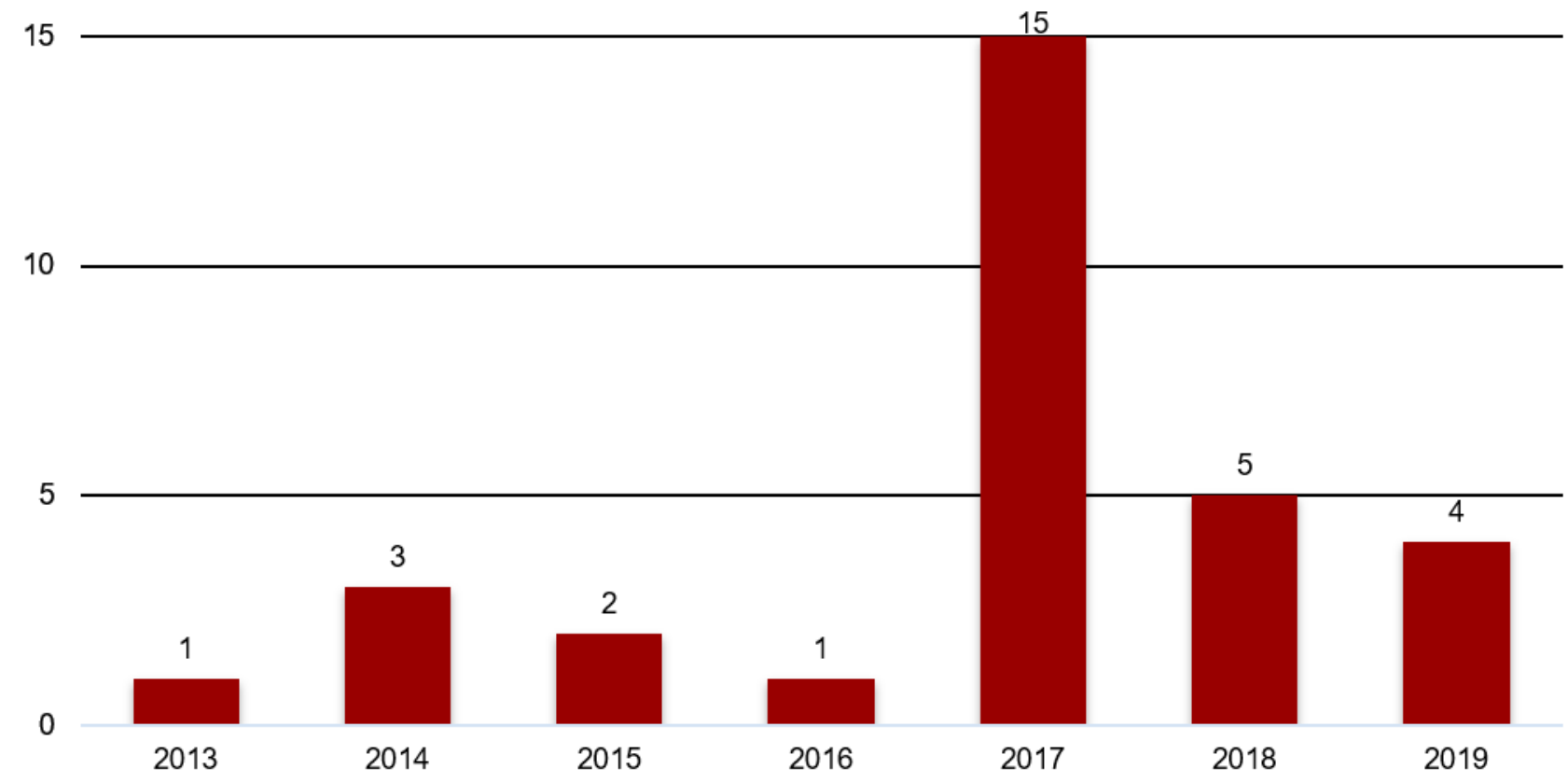

Fonte: os autores. 
Gráfico 3 - Distribuição dos artigos selecionados por público alvo.

14

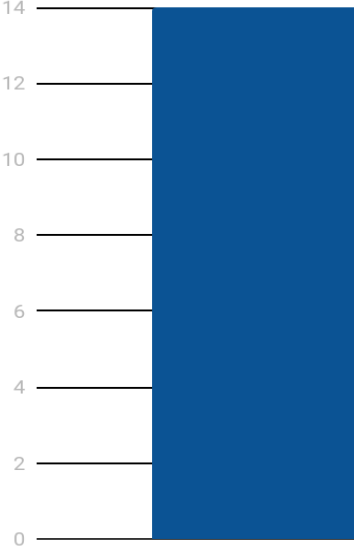

Pacientes

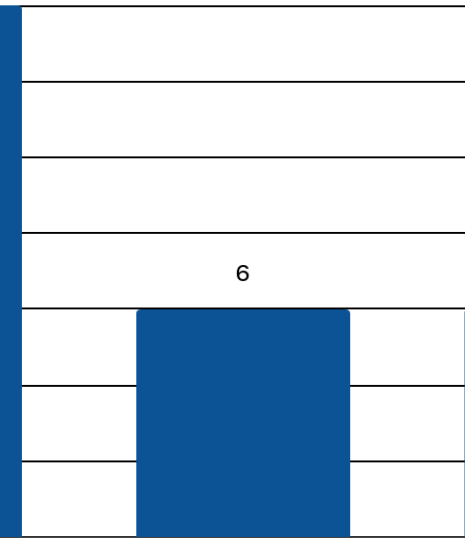

Profissionais
6

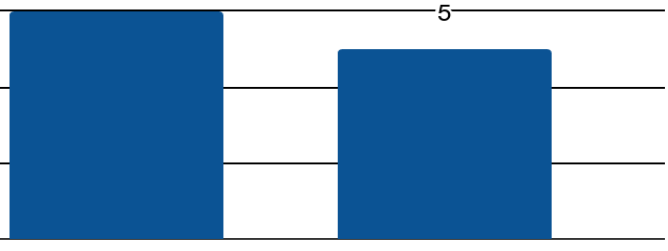

Estudantes

Desenvolvedores

Fonte: os autores.

Gráfico 4 - Assuntos abordados do uso de agentes de inteligência destinados aos pacientes.

- Hábitos de vida

- Educação em saúde

- Adesão ao tratamento

- Prevenção de doenças

- Diagnóstico de doenças

- habilidades sociais

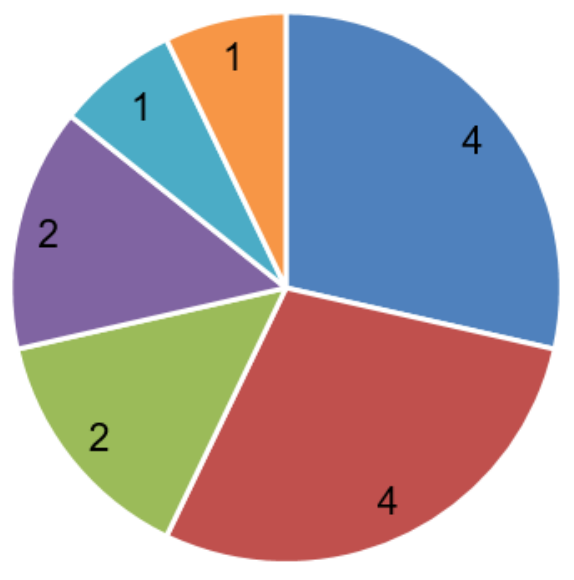

Fonte: os autores.

Gráfico 5: Assuntos abordados do uso de agentes inteligentes destinados a estudantes.

- Treinamento da prática profissional.

- Reflexão e incentivo a pesquisa na área da tecnologia.

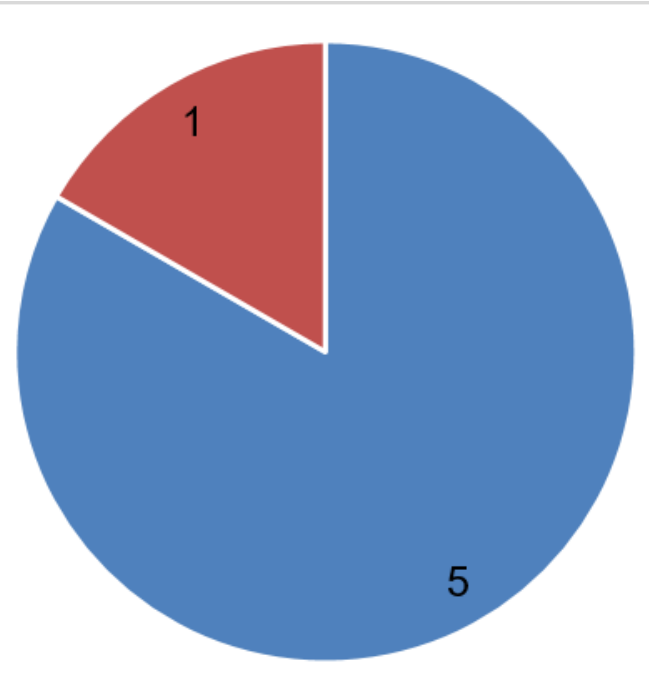

Fonte: os autores.

Rev. Saúde Digital Tec. Educ., Fortaleza, CE, v. 5, n. 1, p.108-122, jan./abr. 2020.

ISSN: 2525-9063 
Gráfico 6: Assuntos abordados do uso de agentes inteligentes destinados a Profissionais.

- Simulação de procedimentos.

- Auxílio nas concultas.

- Avaliação dos chatbots por profissionais.

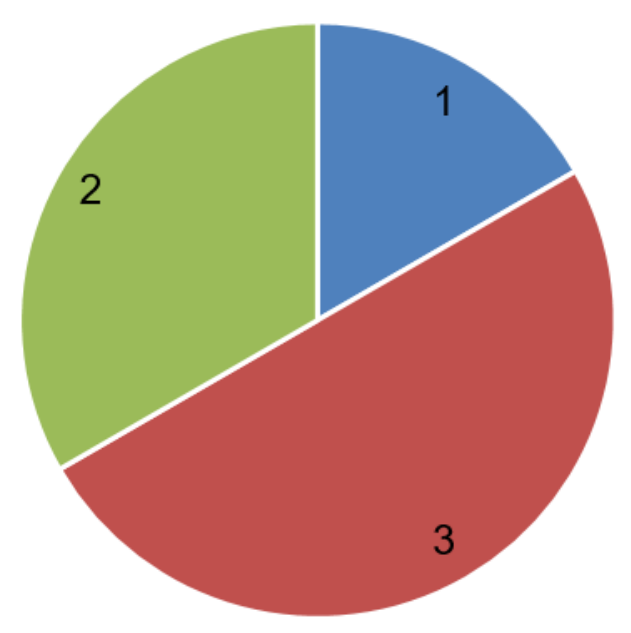

Fonte: os autores.

Gráfico 7 - Assuntos abordados no uso de agentes de inteligência destinados aos desenvolvedores.

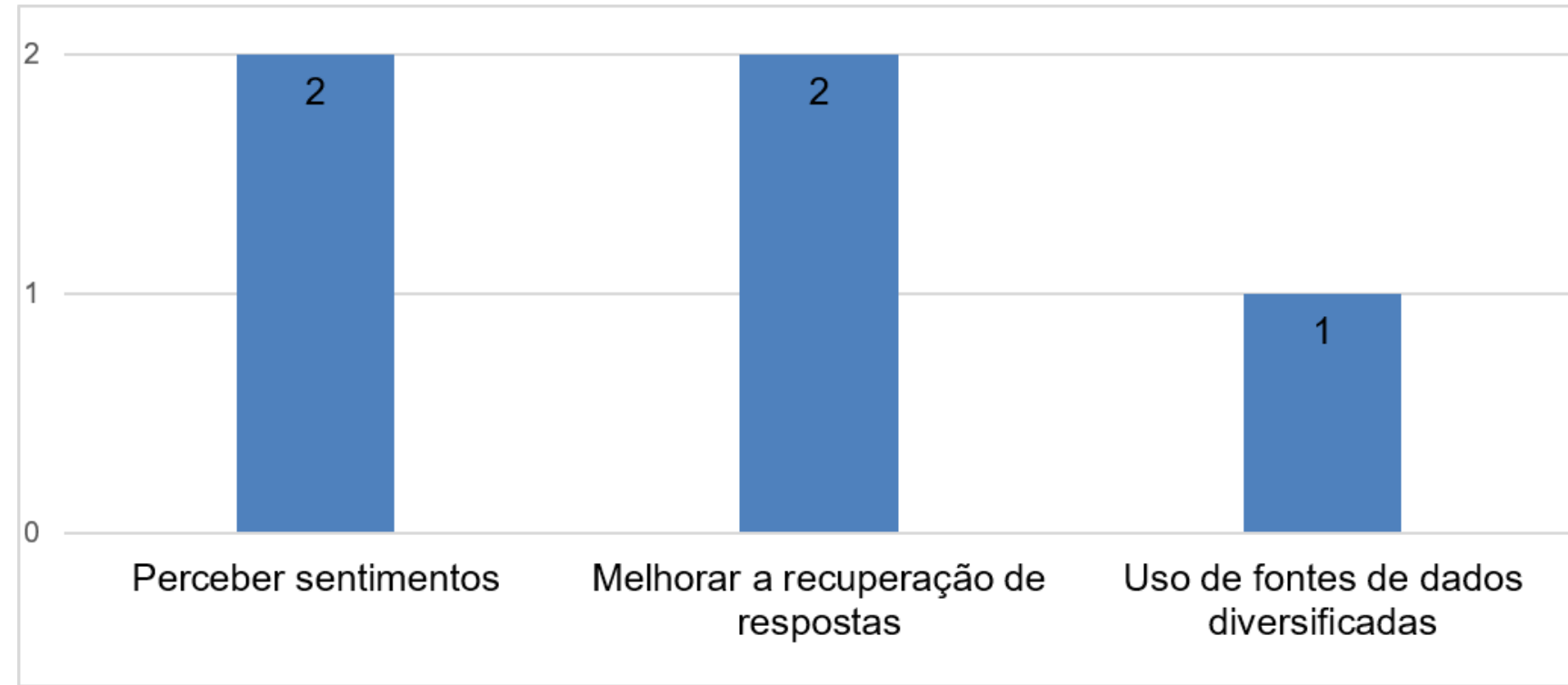

Fonte: Os autores. 


\section{Como citar este artigo}

Cursino, J. R. V. Calista, A.A. Nascimento, J. E. M. Campos Filho, A. S. Uma Revisão Integrativa Sobre o Uso de Chatbot para Subsidiar o Ensino na Área da Saúde Revista de Saúde Digital e Tecnologias Educacionais. [online], volume 5, n. 1. Editor responsável: Luiz Roberto de Oliveira. Fortaleza, mês e ano, p. 108-122. Disponível em: http://periodicos.ufc.br/resdite/index. Acesso em "dia/mês/ano".

Data de recebimento do artigo: 17/11/2019

Data de aprovação do artigo: 25/01/2020

Data de publicação: 17/04/2020 